\title{
Convectons, anticonvectons and multiconvectons in binary fluid convection
}

\author{
ISABEL MERCADER ${ }^{1}$, ORIOL BATISTE ${ }^{1} \dagger$, \\ ARANTXA ALONSO ${ }^{1}$ AND EDGAR KNOBLOCH \\ ${ }^{1}$ Departament de Física Aplicada, Universitat Politècnica de Catalunya, Barcelona 08034, Spain \\ ${ }^{2}$ Department of Physics, University of California, Berkeley, CA 94720, USA
}

(Received 9 April 2010; revised 1 September 2010; accepted 1 September 2010; first published online 13 December 2010)

Binary fluid mixtures with a negative separation ratio heated from below exhibit steady spatially localized states called convectons for supercritical Rayleigh numbers. Numerical continuation is used to compute such states in the presence of both Neumann boundary conditions and no-slip no-flux boundary conditions in the horizontal. In addition to the previously identified convectons, new states referred to as anticonvectons with a void in the centre of the domain, and wall-attached convectons attached to one or other wall are identified. Bound states of convectons and anticonvectons called multiconvecton states are also computed. All these states are located in the so-called snaking or pinning region in the Rayleigh number and may be stable. The results are compared with existing results with periodic boundary conditions.

Key words: bifurcation, buoyancy-driven instability, double diffusive convection

\section{Introduction}

Spatially localized states are of great interest in the theory of pattern formation. They occur not only in vibrating granular media (Umbanhowar, Melo \& Swinney 1996) and polymeric fluids (Lioubashevski et al. 1999) but also in reaction-diffusion systems (Lee et al. 1994), nonlinear optics (Vladimirov et al. 2002), ferrofluids (Richter \& Barashenkov 2005) and in several convection systems, including natural doubly diffusive convection (Ghorayeb \& Mojtabi 1997; Bergeon \& Knobloch 2008), magnetoconvection (Blanchflower 1999; Blanchflower \& Weiss 2002) and binary fluid convection (Batiste \& Knobloch 2005a; Batiste et al. 2006; Jung \& Lücke 2007). Similar structures, localized in the cross-stream direction, are also present in plane Couette flow (Schneider, Gibson \& Burke 2010). Recent years have seen substantial progress in our understanding of such states, both in one and two spatial dimensions. However, this understanding has largely been developed on the basis of simple variational models such as the Swift-Hohenberg equation (Burke \& Knobloch $2007 b$ ). It is therefore of great interest to examine in greater detail the properties of 'real' systems such as convection or shear flows in order to identify gaps in our understanding of the properties of these states, and to suggest extensions of the theory to realistic situations. In the present paper, we focus on spatially localized steady convection in binary mixtures with negative separation ratio heated from below 
(Batiste \& Knobloch 2005a; Batiste et al. 2006), and follow Blanchflower (1999) in referring to such states as convectons to distinguish them from other localized states found in binary fluid convection such as localized travelling waves (Kolodner, Surko \& Williams 1989; Steinberg et al. 1989; Barten et al. 1995; Batiste \& Knobloch $2005 b)$.

In binary fluid convection, convectons have been observed to emerge from dispersive chaos (Kolodner, Glazier \& Williams 1990) via remarkable relaxation oscillations (Batiste et al. 2006): as the Rayleigh number $R$ is increased, the dispersive chaos state undergoes a focusing instability, resulting in spatially localized convection in part of the domain, with convection suppressed elsewhere. When these localized states first begin to form they are weakly unstable and gradually erode before collapsing back into the unstable dispersive chaos state. Thus, the rapid focusing instability is followed by a slow erosion phase before the cycle repeats. Since the cycle involves a chaotic state the resulting oscillation is in general irregular. However, if the footprint excavated in the concentration distribution by the convecton during its lifetime heals on a time scale longer than that of the focusing instability, the convecton will generally reform in the same location, although it may differ from its predecessor. For slightly larger values of $R$ the convectons become stable and do so despite the fact that the background laminar state is unstable to the oscillatory instability responsible for the presence of dispersive chaos at lower $R$. As explained by Batiste et al. (2006), when the oscillatory instability of the conduction state is convective and convectons are present, we do not expect to see a background of travelling waves. Such waves are only observed once the Rayleigh number exceeds the threshold for absolute instability (Alonso et al. 2007).

The binary mixture convectons are organized within a snaking region $R_{-}<R<R_{+}$ in the Rayleigh number $R$. Within this region, one finds two distinct types of convectons, with odd and even parity. The presence of steady distinct parity states can be traced to the presence of a reflection symmetry in the midplane of the system, and so requires not only the Boussinesq symmetry but also identical boundary conditions at the top and bottom of the layer. These states lie on distinct solution branches that snake back and forth across the snaking region. At each saddle-node near $R=R_{-}$, the convecton nucleates a pair of rolls, one on either side, respecting the parity of the structure. As one follows each branch upward, towards $R=R_{+}$, these rolls strengthen to the amplitude of the existing rolls. As a result, successive convectons at $R=R_{+}$ differ by a pair of rolls, one on each side, but are otherwise identical. Thus, the snaking interval contains a large multiplicity of coexisting odd and even convectons of different lengths, many of which turn out to be numerically stable (Batiste et al. 2006; Alonso et al. 2010). The snaking region is a consequence of 'pinning' of the fronts bounding each convecton on either side to the periodic structure within (Pomeau 1986). In analogy with the one-dimensional Swift-Hohenberg equation, we believe that the detailed structure of the resulting snaking or pinning region (Burke \& Knobloch $2007 \mathrm{~b}$ ) is a consequence of the transverse intersections of the unstable manifold of the conduction state and the centre-stable manifold of periodic convection within the steady-state convection equations viewed as a dynamical system in space (Woods \& Champneys 1999; Coullet, Riera \& Tresser 2000; Beck et al. 2009). The necessary conditions for its presence are $(a)$ coexistence between the conduction and convection states (i.e. the requirement that convection be subcritical), (b) spatial reversibility (i.e. the requirement that the equations describing steady convection are invariant under reflection $x \rightarrow-x$, together with the corresponding change in the dependent variables) and $(c)$ the presence of a heteroclinic connection between the conduction 
(a)

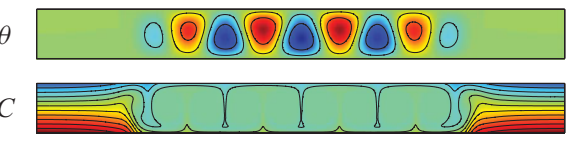

(b)

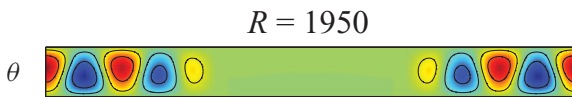

C

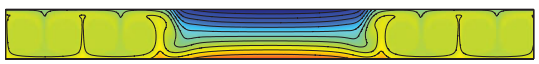

(c)

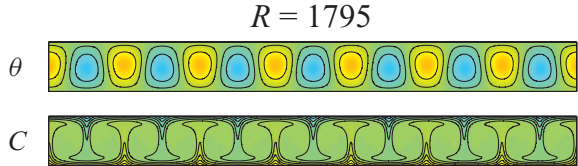

$(d)$

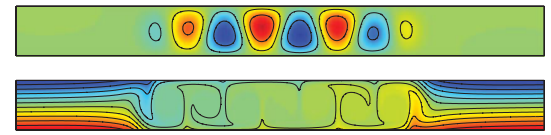

(e)

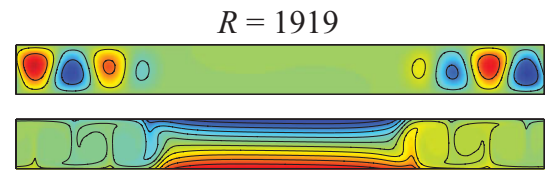

$(f)$

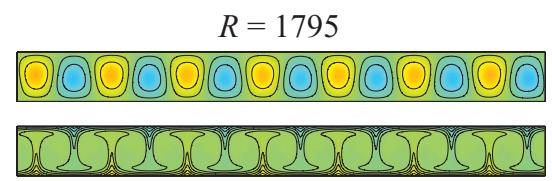

FiguRE 1. (Colour online) ( $a$ ) An even-parity convecton and $(b)$ a phase-matched even-parity anticonvecton, both of which terminate together on an even-parity $\mathrm{P}_{7}$ state with seven roll pairs within the $\Gamma=14$ periodic domain $(c)$. $(d)$ An odd-parity convecton and $(e)$ a phase-matched odd-parity anticonvecton, both of which terminate together on an odd-parity $\mathrm{P}_{7}$ state $(f)$. This state is a translate of the state $(c)$, implying that both even and odd localized states terminate at the same Rayleigh number, here $R=1795$. The states are visualized in terms of contours of constant temperature fluctuation $\theta$ (upper panels) and contours of constant concentration $C$ (lower panels). Here and elsewhere, all solution profiles use the same colour table to indicate the amplitude of the temperature and concentration fields. From Mercader et al. (2010a).

and convection states (i.e. the presence of a front connecting these states). Under these circumstances, reversibility guarantees the existence of a reverse front and these fronts can then be used back-to-back to construct different types of steady localized structures provided $R$ is confined to the pinning region (Champneys 1998; Hunt et al. 2000; Burke \& Knobloch 2007b).

Binary fluid convection is subcritical for sufficiently negative separation ratios $S$. Existing computations of the convectons present in this case used periodic boundary conditions (PBC) and a value of $S$ for which the snaking or pinning region was quite narrow (Batiste et al. 2006). For larger values of $|S|$, the width of this interval increases (Mercader et al. 2010b) and for the parameters used below convectons are present over a relatively wide interval of Rayleigh numbers, allowing us to study the variety of steady states present in the snaking region. For this purpose, we use a numerical domain with a modest aspect ratio $\Gamma$. Since the process of nucleating new rolls must terminate when the domain is full, snaking in a finite domain terminates after a finite number of turns. The manner in which this occurs depends strongly on the boundary conditions used and differs profoundly between PBC (Bergeon et al. 2008) and closed container boundary conditions (Mercader et al. 2009). With PBC, the bifurcation diagram shows a pair of branches of localized states with odd and even parity. These bifurcate together from the subcritical primary branch of periodic states and typically (but not always) terminate together in an Eckhaus bifurcation on another branch of periodic states. For the purposes of this paper, it is important to understand in detail what occurs at this bifurcation. This understanding is complicated by the presence of midplane reflection symmetry whenever the boundary conditions at top and bottom of the layer are identical (the usual case). Figure 1(a) shows a typical even-parity convecton in a spatially periodic domain with period $\Gamma=14$ computed by Mercader et al. (2010a). The convecton has been placed at the centre of the domain although it can of course form anywhere in the domain. In particular, if the convecton is 
translated by $\Gamma / 2$ the void region, hereafter an 'anticonvecton', will be located in the centre of the domain instead of the convecton itself. The original convecton now appears split into two portions, with half located at the left boundary and half at the right boundary. Figure $1(b)$ shows an anticonvecton obtained from figure $1(a)$ by a $\Gamma / 2$ translation, followed by a reflection in the midplane. The convecton and anticonvecton generated in this way are not only both reflection-symmetric with respect to $x=0$ but are in addition phase-matched, in the sense that the rising and descending plumes are located at the same spatial locations in both. As a result, both bifurcate simultaneously from the even-parity periodic state shown in figure $1(c)$. As shown in figure $1(d-f)$ the same construction for odd-parity convectons shows that the odd-parity convecton shown in figure $1(d)$ and the odd-parity anticonvecton in figure 1(e) bifurcate simultaneously from the odd-parity periodic state in figure $1(f)$. Moreover, since each of the states in figure 1 can be reflected in the midplane to obtain new solutions, there are in fact eight branches of localized states in the bifurcation diagram, all of which bifurcate simultaneously from a circle of periodic states and all of which terminate simultaneously on another circle of periodic states (Mercader et al. 2010a). However, since the four even-parity states are related by symmetry, they appear as a single branch in a bifurcation diagram that shows the Nusselt number as a function of the Rayleigh number, and similarly for the odd-parity states. The circle of periodic states is likewise represented by a single branch (with all translationrelated periodic states represented by a single representative). Such a representation allows for the fact that the localized states terminate on different representatives from the group orbit of these states (figure 1). These observations are important in what follows since they allow us to understand the consequences of changing the lateral boundary conditions away from PBC.

In fact, the odd- and even-parity states do not always terminate together on the same branch of periodic states - their termination points change discontinuously as $\Gamma$ varies through a process that resembles 'unzipping' (Bergeon et al. 2008; Dawes 2009), and occurs at different critical values of $\Gamma$ for different parity states. In cases where the branches of localized states terminate on different branches of periodic states, each termination point is also a termination point of a branch of mixed modes that does not snake (Bergeon et al. 2008; Mercader et al. 2009). Thus, in all cases the Eckhaus point is the termination point of two branches, one of which consists of states with odd parity while the other consists of states with even parity. Once again this situation corresponds to the simultaneous termination of eight branches, four of which correspond to even states that terminate on an even-parity periodic state while the remaining four correspond to odd states that terminate on an odd-parity periodic state, as seen in figure 1.

This paper is motivated by a simple question: do the convecton and anticonvecton states persist when the boundary conditions are changed to realistic lateral boundary conditions and do they retain their properties? It is immediately clear that the lateral boundaries destroy the symmetry relating the convecton and anticonvecton states by eliminating the translation invariance that relates them. Thus, one expects the convecton and anticonvectons to have different properties, even if they persist in some form or other. In this paper we show that these states are indeed present in domains with realistic lateral boundary conditions and show how their properties are modified by the presence of the boundaries. We find it easiest to consider the case of Neumann boundary conditions (NBC) first. These correspond to free-slip thermally insulating boundary conditions. Once we understand the role played by the lost symmetry in this case, we can understand the origin of the more complex states 
we find with realistic closed container boundary conditions. These include not only anticonvectons but also convectons attached to one or other lateral boundary, and a variety of bound states of two convectons. We show by numerical continuation that all these states snake in a particular interval of Rayleigh numbers called the snaking or pinning region, and relate the results to existing results with $\mathrm{PBC}$.

This paper is organized as follows. In $\S 2$, we introduce the equations for binary fluid convection, followed in $\S 3$ by a description of our results on single-pulse localized states with NBC. These results are extended to closed containers with no-slip boundaries in $\S 4$. Sections 5 and 6 describe the corresponding results for two-pulse states. The paper concludes with a brief discussion in $\S 7$.

\section{Formulation of the problem}

Binary mixtures are characterized by cross-diffusion quantified by the separation ratio $S$. When $S<0$, the heavier component (of concentration $C$ ) migrates up the temperature gradient. Thus, in a layer heated from below the destabilizing temperature gradient competes with a stabilizing concentration gradient that develops in response to the heating. If this effect is strong enough convection sets in as growing oscillations once the Rayleigh number $R$ exceeds a critical value $R_{c}$; this instability is typically subcritical and develops into a variety of travelling wave states depending on parameters and initial conditions. The convectons that are of interest here emerge from this time-dependent state as described by Batiste et al. (2006).

In this paper we study binary fluid convection in two spatial dimensions. The system is described by the dimensionless equations (Mercader, Alonso \& Batiste 2004)

$$
\begin{aligned}
\boldsymbol{u}_{t}+(\boldsymbol{u} \cdot \nabla) \boldsymbol{u} & =-\nabla P+\sigma R[(1+S) \theta-S \eta] \hat{z}+\sigma \nabla^{2} \boldsymbol{u}, \\
\theta_{t}+(\boldsymbol{u} \cdot \nabla) \theta & =w+\nabla^{2} \theta \\
\eta_{t}+(\boldsymbol{u} \cdot \nabla) \eta & =\tau \nabla^{2} \eta+\nabla^{2} \theta
\end{aligned}
$$

together with the incompressibility condition

$$
\nabla \cdot \boldsymbol{u}=0 .
$$

Here $\boldsymbol{u} \equiv(u, w)$ is the velocity field in $(x, z)$ coordinates, $P$ is the pressure, and $\theta$ denotes the departure of the temperature from its conduction profile, in units of the imposed temperature difference $\Delta T>0$ across the layer. The variable $\eta$ is defined such that its gradient represents the dimensionless convective mass flux. Thus, $\eta \equiv \theta-\Sigma$, where $C=1-z+\Sigma$ is the concentration of the heavier component in units of the concentration difference that develops across the layer as a result of crossdiffusion. The system is specified by four dimensionless parameters: the Rayleigh number $R$ providing a dimensionless measure of the imposed temperature difference $\Delta T$, the separation ratio $S$ that measures the resulting concentration contribution to the buoyancy force due to cross-diffusion, and the Prandtl and Lewis numbers $\sigma$, $\tau$, in addition to the aspect ratio $\Gamma$. For the no-slip fixed temperature boundaries employed here $\boldsymbol{u}=\theta=\eta_{z}=0$ on $z=0,1$.

In the following, we shall be interested in different types of steady solutions of (2.1)(2.4). Of particular interest are even- and odd-parity solutions. These satisfy $(u(x, z)$, $w(x, z), \theta(x, z), \eta(x, z))=(-u(-x, z), w(-x, z), \theta(-x, z), \eta(-x, z))$ and $(u(x, z)$, $w(x, z), \theta(x, z), \eta(x, z))=-(u(-x, 1-z), w(-x, 1-z), \theta(-x, 1-z), \eta(-x, 1-z))$, respectively, relative to a suitable origin in $x$. Thus, even solutions are invariant under the reflection $x \rightarrow-x$ while odd states are point-symmetric. These symmetries in turn 
imply that both states are stationary and hence do not drift. These are the only steady solutions that can bifurcate from the conduction state (Crawford \& Knobloch 1991). We compute such solutions using numerical continuation as described by Mercader, Batiste \& Alonso (2006). A Newton solver together with initial conditions of appropriate type is used to find steady solutions required to initialize the procedure. Throughout we focus on domains of aspect ratio $\Gamma=14$ subject to one of three sets of boundary conditions in the horizontal: (i) $\mathrm{PBC}$ with period $\Gamma$, (ii) $\mathrm{NBC}$, viz.

$$
u=w_{x}=\theta_{x}=\eta_{x}=0 \quad \text { on } \quad x= \pm \Gamma / 2,
$$

and (iii) insulating closed container boundary conditions (ICCBC), viz.

$$
\boldsymbol{u}=\theta_{x}=\eta_{x}=0 \quad \text { on } \quad x= \pm \Gamma / 2,
$$

corresponding to no-slip, thermally insulating lateral boundaries. The modest aspect ratio used is large enough to allow well-localized structures and permits a much more detailed study of the problem than the aspect ratio $\Gamma=60$ used by Batiste et al. (2006).

The results obtained below are for water-ethanol mixtures with $S=-0.1, \sigma=7$, $\tau=0.01$. For these parameter values, the conduction state loses stability at a Hopf bifurcation at $R_{c} \approx 1909$; however, we do not compute the time-dependent states that result.

\section{Single-pulse states: Neumann boundary conditions}

NBC have a simple but very useful property. All steady solutions satisfying NBC on a domain of aspect ratio $\Gamma$ can be found by solving the problem on a periodic domain with period $2 \Gamma$ and picking out the solutions that solve the original NBC problem (Crawford et al. 1991). The essential point is that any solution of the NBC problem can be reflected in a lateral boundary without introducing discontinuities in any spatial derivative; as a result, the solution with its reflection satisfies the equation on the domain $2 \Gamma$ with PBC. Moreover, all solutions of this PBC problem that are even with respect to the boundary automatically satisfy $\mathrm{NBC}$ on the domain $\Gamma$. This simple observation has several consequences. First, the linear stability problem for the conduction state is solved by sines and cosines with fixed values of the mode number. These eigenstates can be used to construct nonlinear states and these can therefore be characterized by the mode number of the eigenstate. Thus even solutions have wavenumbers $2 \pi n / \Gamma$ (plus harmonics) while odd solutions have wavenumbers $2 \pi(n+(1 / 2)) / \Gamma$ (plus harmonics), where $n$ is an integer $(n=0,1, \ldots)$. This is not the case for non-NBC for which the linear eigenstates are not pure sines or cosines, and the wavelength of the solution can change as a function of its amplitude.

The fact that the NBC problem can be embedded in a PBC problem is responsible for the presence of a 'hidden' translation symmetry in the NBC problem (Crawford et al. 1991), which is in turn responsible for the presence of well-defined mode numbers. This hidden symmetry is also responsible for the fact that not only oddparity but also even-parity solutions bifurcate from the conduction state in pitchfork bifurcations.

\subsection{Even-parity convectons and anticonvectons}

The bifurcation diagram showing the Nusselt number $N u$ for even-parity convectons with NBC as a function of the Rayleigh number $R$ is shown in figure $2(a)$. These states are centred in the middle of the domain (figure $3 a$, top) and snake within a snaking 

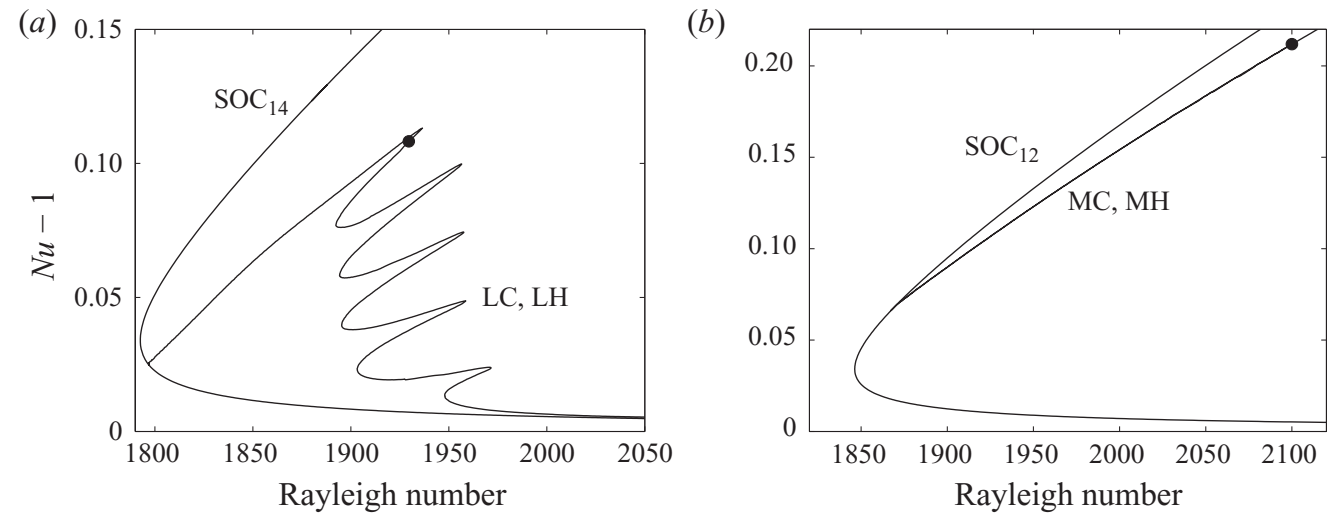

FIGURE 2. Bifurcation diagrams with $\mathrm{NBC}$ on a $\Gamma=14$ domain showing $(a)$ even-parity localized convectons (LC) and phase-matched anticonvectons (LH), and (b) even-parity phase-matched mixed modes of convecton (MC) and hole (MH) type. The LC and LH states are related by a 'hidden' symmetry and likewise for the mixed modes, and so are represented by a single branch. The LC/LH branch snakes and the width of the snaking region is identical to that of even-parity states with $\mathrm{PBC}$. Once the domain is almost filled, the branch exits the snaking region to the left and terminates on the branch $\mathrm{SOC}_{14}$ of 14 rolls within the domain $\Gamma$. The mixed modes bifurcate together from the branch $\mathrm{SOC}_{12}$ of 12 rolls within $\Gamma$ and do not snake. The solid dots indicate the location of the solutions shown in figure 3 .

(a)

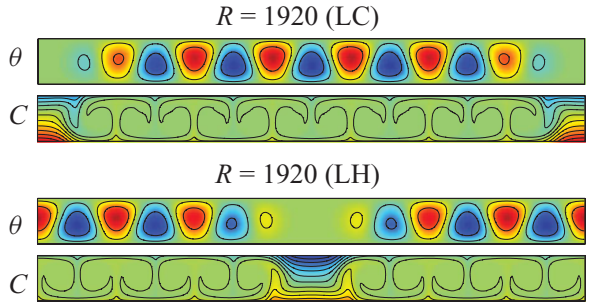

(b)

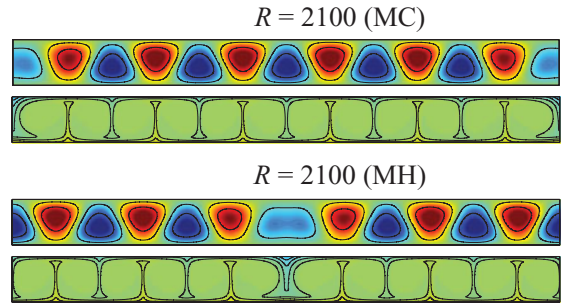

FIGURE 3. (Colour online) Even-parity states with NBC in a $\Gamma=14$ domain. (a) Top: an even-parity convecton (LC). Bottom: a phase-matched even-parity anticonvecton (LH), both at $R=1920$. The two states are related by a 'hidden' symmetry and so correspond to a single location (solid dot) in the bifurcation diagram in figure $2(a)$. The states terminate together on the branch $\mathrm{SOC}_{14}$. (b) Top: an even-parity mixed mode of C-type (MC). Bottom: a phase-matched even-parity mixed mode of H-type (MH), both at $R=2100$. The two states are related by a 'hidden' symmetry and so correspond to a single location (solid dot) in the bifurcation diagram in figure $2(b)$. The states terminate together on the branch $\mathrm{SOC}_{12}$.

or 'pinning' region that is identical to the corresponding region with $\mathrm{PBC}$. The figure shows, in addition, the branch of phase-matched even-parity anticonvectons or 'hole' states with the hole centred in the middle of the domain (figure $3 a$, bottom). In the following, we refer to these states as localized convectons (LC) and localized holes (LH), respectively. Both states carry the same heat flux and hence are represented by identical curves in the bifurcation diagram. This is a consequence of the fact that both solve the same PBC problem with period $2 \Gamma$ and hence are related by a 'hidden' translation symmetry (Crawford et al. 1991). When the domain is almost full, the two branches exit the snaking region and terminate together on a branch $\mathrm{SOC}_{14}$ consisting of 14 rolls within the domain. This state is a periodic solution of the PBC problem with $\Gamma=14$, and since it contains seven full wavelengths within $\Gamma$ we also refer to it as $\mathrm{P}_{7}$. 
(a)
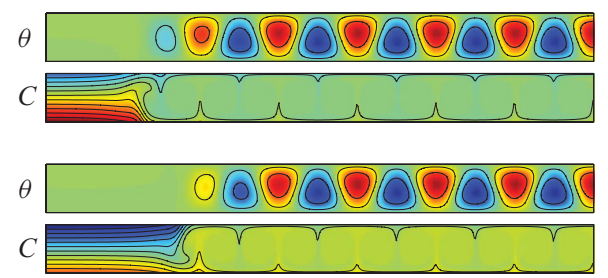

(b)

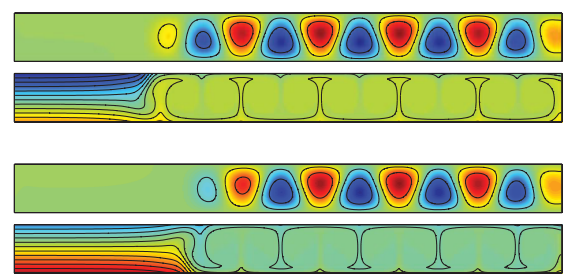

FIGURE 4. (Colour online) Different localized wall (LW) states in a $\Gamma=14$ domain with $(a)$ NBC, both at $R=1930$, and $(b)$ ICCBC, both at $R=1957$. The location of the states on the associated LW branches is indicated in figure $5(a, b)$ by solid dots.

Figure 2(b) shows a different class of states allowed by NBC called mixed modes. These states also bifurcate from spatially periodic solutions (PBC case) or from the corresponding SOC solutions (NBC case) but do not snake. The mixed modes represent spatially extended convection with superposed amplitude modulation with maximum permitted wavelength. These states are shown in figure $3(b)$. The top panel shows a mixed mode with minimum amplitude at the walls (hereafter, C-type mixed mode), while the bottom panel shows a mixed mode with minimum amplitude in the centre (hereafter H-type mixed mode). In the following, we think of the mixed modes as representing extended states with a defect in an otherwise periodic structure. Like the convecton and hole states, these two defect states are related by a hidden translation symmetry and hence fall on identical curves in the bifurcation diagram (figure $2 b$ ). Both solutions bifurcate together from the branch $\mathrm{SOC}_{12}$ of 12 rolls within the domain $\Gamma$; this branch is an example of a subsidiary primary periodic branch and may be labelled $\mathrm{P}_{6}$. Mixed modes play a prominent role in our discussion of odd-parity localized states, as discussed next.

\subsection{Localized wall states}

The hole state in figure 3( $a$, bottom) resembles a pair of wall states, with one attached to either lateral wall. Figure 2(a) shows that these wall-attached states snake in the classic fashion. However, as shown in figure 4(a), we can also find solutions with a wall state attached to just one of the lateral walls. We refer to states of this type as localized wall states or LW for short. Since we impose NBC at the wall, this solution in fact represents an even convecton on a domain of length $2 \Gamma$. As a result, it snakes as it would do in a $2 \Gamma$ domain, implying that with each oscillation of the branch a single roll is added at the left as the structure grows away from the boundary. Thus, the branch undergoes twice as many back and forth excursions (figure $5 a$, dashed line) as the LC branch (figure $5 a$, solid line, reproduced from figure $2 a$ ) before the domain is full and the branch exits the snaking region, and terminates on the $\mathrm{SOC}_{14}$ branch. Moreover, since the effective domain is $2 \Gamma$, the $\mathrm{LW}$ branch terminates closer to the saddle-node than the LC branch (Bergeon et al. 2008).

\subsection{Odd-parity convectons and anticonvectons}

We now turn to the more interesting case of odd-parity convectons. In figure $6(a)$ we show a branch of centred convectons LC and a branch of mixed modes labelled $\mathrm{M}_{13}$. As in the case of even-parity states, the former snake while the latter do not. The two branches terminate together on a branch labelled $\mathrm{SOC}_{13}$ with 13 rolls in the NBC domain, with the result that the diagram resembles similar diagrams obtained with PBC. However, in the PBC case the two (distinct) branches that terminate together on a periodic branch correspond to states of opposite parity (one is odd while the other 

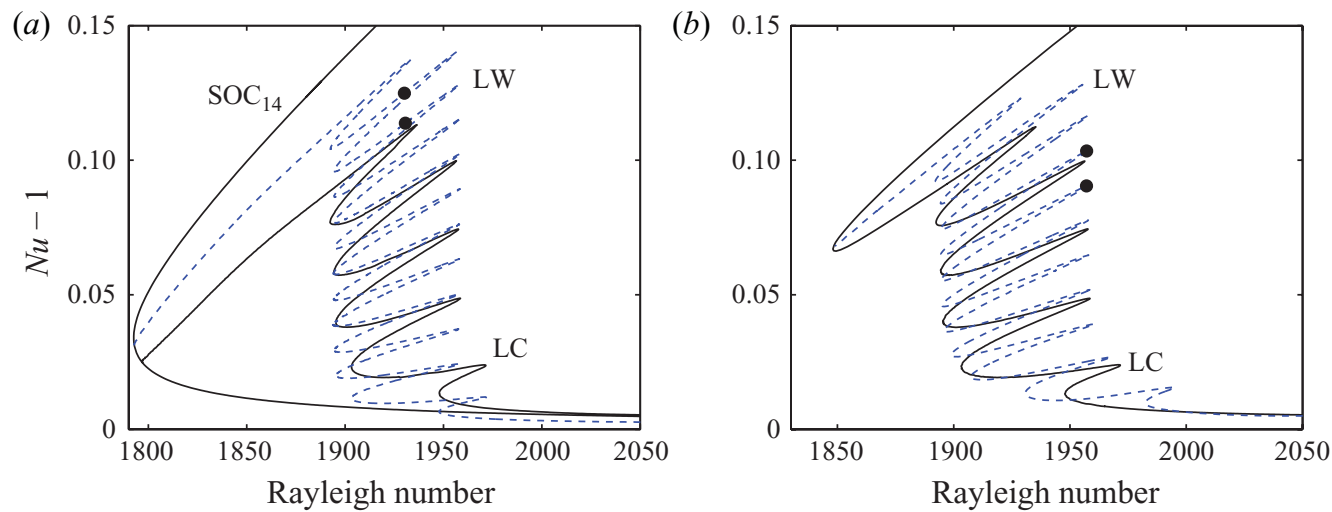

Figure 5. (Colour online) Bifurcation diagrams for localized wall (LW, dashed line) states and single-pulse convectons (LC, solid line) for $(a) \mathrm{NBC}$ and (b) ICCBC in a $\Gamma=14$ domain. The wall states differ by one roll at successive right saddle-nodes, and hence require twice as many turns to fill the domain as a localized convecton or hole. The solid dots indicate the location of the solutions shown in figure $4(a, b)$.
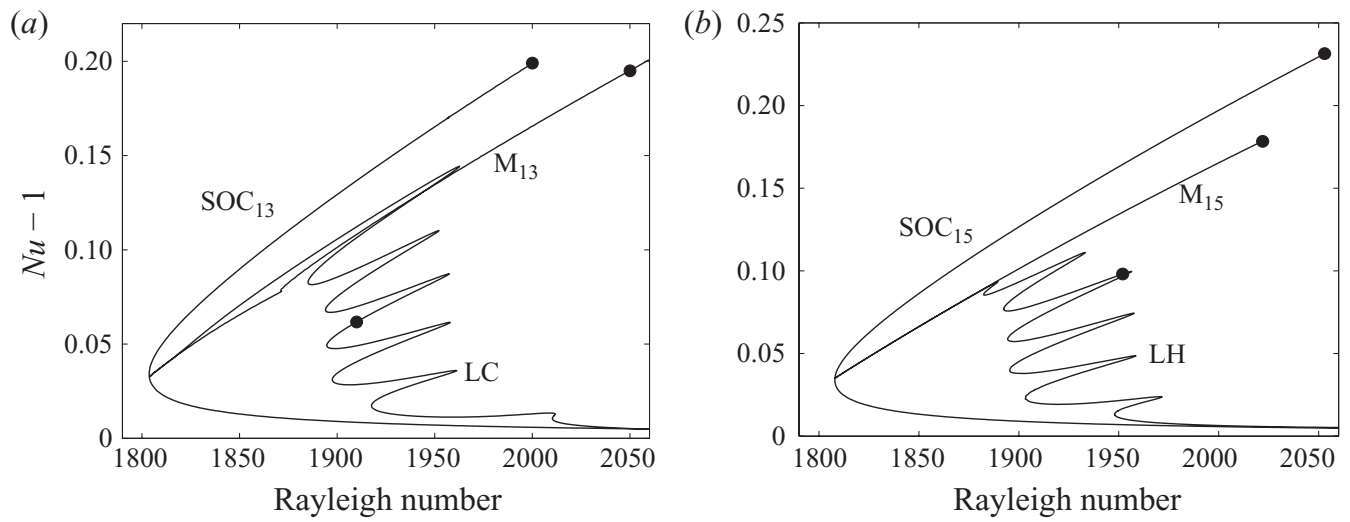

FIGURE 6. Bifurcation diagrams with $\mathrm{NBC}$ on a $\Gamma=14$ domain showing $(a)$ odd-parity localized convectons LC and $(b)$ odd-parity anticonvectons LH. The LC terminate on the branch $\mathrm{SOC}_{13}$ consisting of 13 rolls in the $\Gamma=14$ domain, together with a branch of H-type mixed modes $\mathrm{M}_{13}$. The $\mathrm{LH}$ terminate on the branch $\mathrm{SOC}_{15}$ together with a branch of C-type mixed modes $\mathrm{M}_{15}$. Examples of each solution type are shown in figure 7 . The width of the LC and LH snaking regions is the same as that for even-parity LC.

is even) while here both branches correspond to odd-parity states. These are shown in figure 7( $a$, top and middle) and terminate together on an odd-parity periodic state (bottom), just as the even-parity states terminate together on an even-parity periodic state. Since this (Eckhaus) bifurcation necessarily creates modulated states of C- and $\mathrm{H}$-types, the mixed modes are necessarily of $\mathrm{H}$-type (figure $7 a$, middle).

Figure 6(b) shows the corresponding results for centred odd-parity anticonvectons. This time it is the anticonvecton branch LH that snakes but it terminates on the branch $\mathrm{SOC}_{15}$ with 15 rolls in the domain and does so simultaneously with an oddparity mixed-mode branch $\mathrm{M}_{15}$ with 15 rolls that does not snake. The states on the latter are necessarily of C-type, i.e. the defect is now 'divided' between the left and right boundaries so that the solution resembles a domain-filling convecton. The $\mathrm{LH}$ 

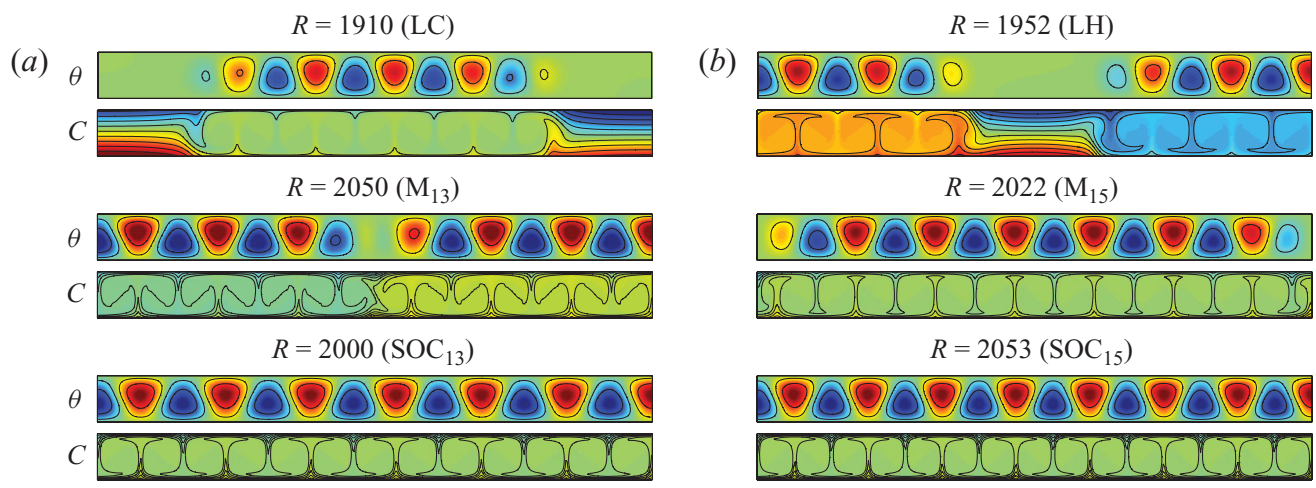

Figure 7. (Colour online) Odd-parity states with $\mathrm{NBC}$ in a $\Gamma=14$ domain. (a) Top: an odd-parity convecton (LC). Middle: an odd-parity mixed-mode state of H-type $\left(\mathrm{M}_{13}\right)$. Bottom: an odd-parity $\mathrm{SOC}_{13}$ state. The states $\mathrm{LC}$ and $\mathrm{M}_{13}$ terminate together on $\mathrm{SOC}_{13}$ (figure $6 a$ ). $(b$ ) Top: an odd-parity anticonvecton (LH). Middle: an odd-parity mixed-mode state of C-type $\left(\mathrm{M}_{15}\right)$. Bottom: an odd-parity $\mathrm{SOC}_{15}$ state. The states $\mathrm{LH}$ and $\mathrm{M}_{15}$ terminate together on $\mathrm{SOC}_{15}$ (figure $6 b$ ). Neither $\mathrm{SOC}_{13}$ nor $\mathrm{SOC}_{15}$ solves the PBC problem with $\Gamma=14$ but each solves the PBC problem with period $2 \Gamma$. The mixed-mode states $\mathrm{M}_{13}$ and $\mathrm{M}_{15}$ resemble odd-parity hole and convecton states, respectively, but do not snake. The disparity in the average concentration on either side of the holes in the $\mathrm{LH}$ and $\mathrm{M}_{13}$ states is maintained through concentration pumping by the rolls on either side of the hole, together with no-flux boundary conditions at $x= \pm \Gamma / 2$.

and $\mathrm{M}_{15}$ states are shown in figure $7(b$, top and middle) and once again terminate on an odd-parity periodic state, this time $\mathrm{SOC}_{15}$ (bottom).

The superposition of the bifurcation diagrams in figure $2(a, b)$ and figure $6(a, b)$ can be thought of as the result of an unfolding of the bifurcation diagram with PBC with its eight branches of localized states. This unfolding is a consequence of changing the PBC to NBC and splits apart the even- and odd-parity branches, and in addition the odd-parity convectons and odd-parity anticonvectons. Because of the absence of translation invariance, each of these states now terminates on a different branch of periodic states. This behaviour in turn demands the presence of mixed-mode states; in fact, these are present not only in the NBC case (figures $2 b$ and $6 a, b$ ) but in the PBC case as well (Mercader et al. 2010a). Thus, the loss of translation invariance has a dramatic effect on the bifurcation diagram with PBC.

\section{Single-pulse states: closed container boundary conditions}

In this section, we examine the corresponding states with the ICCBC. As noted by Mercader et al. (2009), see also Houghton \& Knobloch (2009) and Kozyreff, Assemat $\&$ Chapman (2009), the presence of non-Neumann boundary conditions destroys the periodic state present with PBC and its counterpart in the NBC problem. As a result, the snaking branches can no longer terminate on a periodic branch, and one instead finds that as the domain fills up with convection rolls the snaking branches leave the snaking region in the direction of larger Rayleigh numbers and turn continuously into a large amplitude state resembling the mixed modes $M$ present with $\mathrm{PBC}$ and NBC. Mercader et al. (2009) refer to this situation as snaking without bistability. Figure 8 shows the resulting bifurcation diagrams for both even and odd states. Figure $9(a)$ shows an even-parity convecton (bottom), together with its large-amplitude domainfilling counterpart (top). The former is very similar to the corresponding NBC state, 

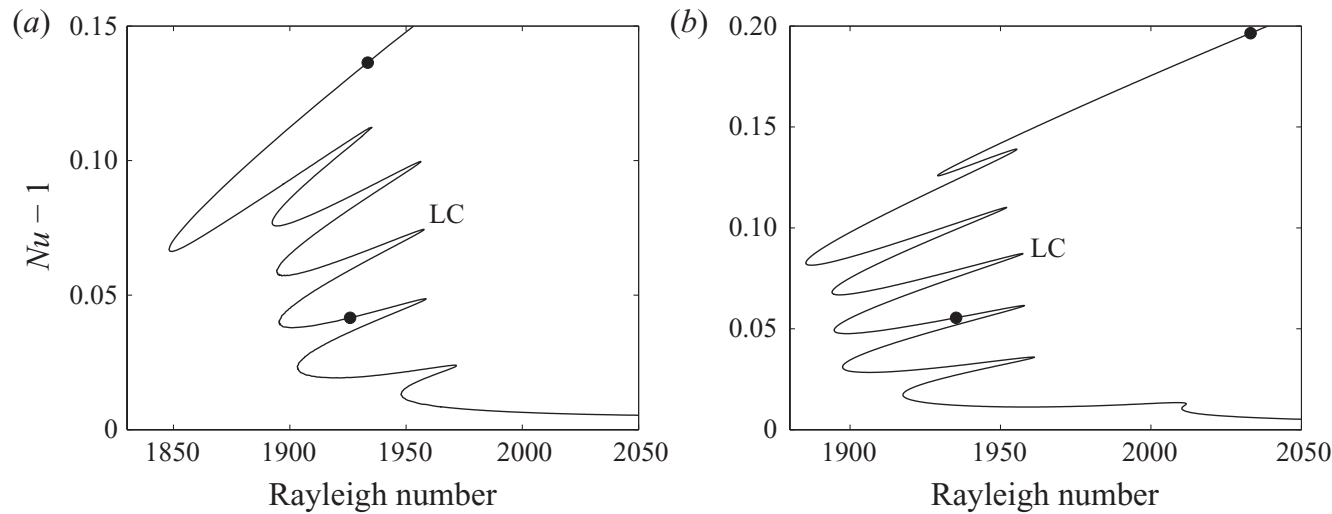

FIGURE 8. Bifurcation diagrams for $(a)$ even and $(b)$ odd-parity centred convectons with ICCBC in a $\Gamma=14$ domain. Solid dots indicate location of the states shown in figure 9. Both convecton branches turn continuously into large amplitude branches of domain-filling states with defects at either boundary (figure 9).

(a)

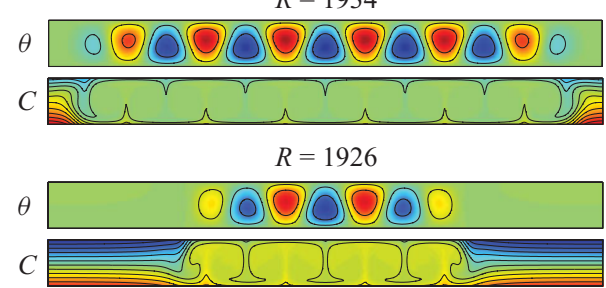

(b)

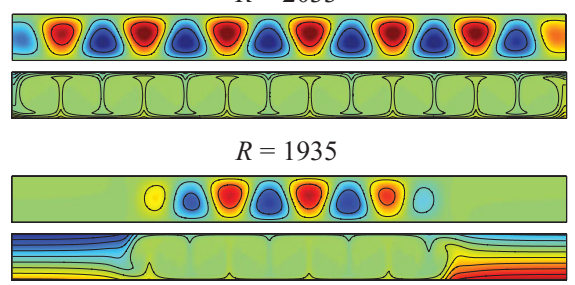

Figure 9. (Colour online) Convectons with ICCBC in a $\Gamma=14$ domain at locations indicated by solid dots in figure 8. (a) Even-parity convectons at $R=1934$ (top) and $R=1926$ (bottom). (b) Odd-parity convectons at $R=2033$ (top) and $R=1935$ (bottom).

since the lateral walls are far from the structure. However, the extended state shown in the top panel reveals the effect of the lateral walls, which reduce the flow in their vicinity suppressing advection. The corresponding results for odd convectons are shown in figure $9(b)$. Anticonvectons consisting of a pair of wall-attached states with a hole in between can also be found but are affected more strongly by the boundary conditions (see $\S 6$ ).

As shown in figure $5(b)$, we can also find wall-attached states analogous to the state shown in figure 5(a) computed with NBC. Figure 4(b) shows sample solutions. Except for the defect at the right wall, the structure snakes exactly as in the NBC case, although this time when the wall-attached state becomes so large that it fills the domain it connects to the branch LC of even convectons at a secondary bifurcation, i.e. it turns into an even state after exiting the snaking region (figure $5 b$ ).

\section{Two-convecton states: Neumann boundary conditions}

As is well-known from the study of localized states in the Swift-Hohenberg equation (Wadee, Coman \& Bassom 2002; Burke \& Knobloch 2009; Knobloch et al. 2010) and related systems (van der Heijden, Champneys \& Thompson 2002), the pinning or snaking region also contains multipulse states, i.e. bound states of two or more convectons. With PBC, two identical and equidistant localized states are equivalent to a single-pulse state on half the domain. Thus, equidistant two-pulse convectons 

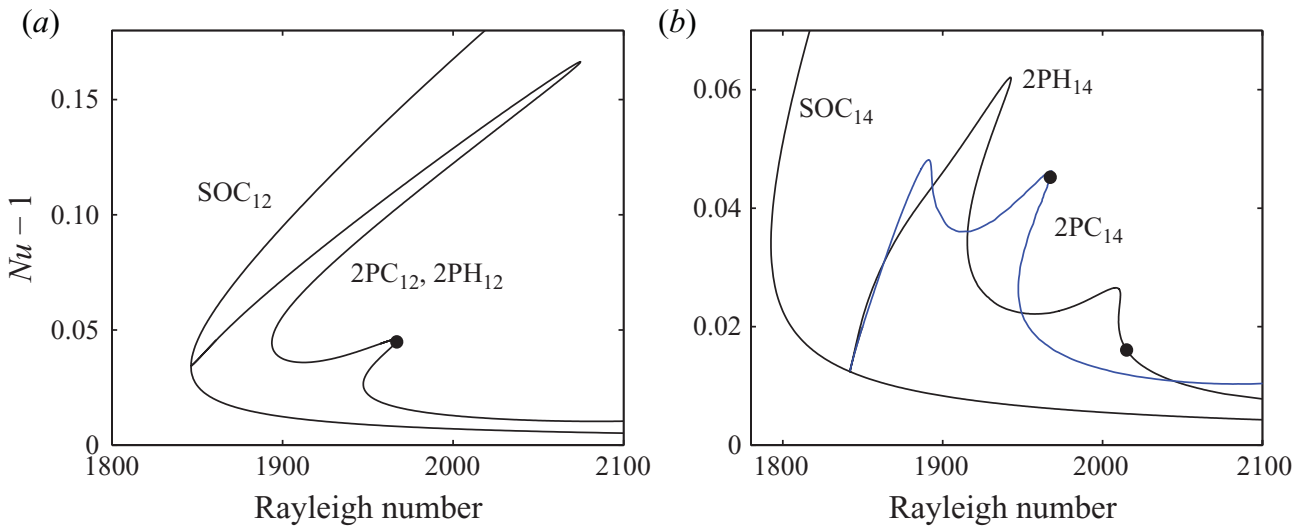

FiguRE 10. (Colour online) Bifurcation diagrams for even-parity two-pulse states in a $\Gamma=14$ domain. (a) Solutions based on 12 rolls. (b) Solutions based on 14 rolls. Solid dots indicate the location of the solutions shown in figure 11.

should also snake, and execute half as many turns as a single convecton, before filling the domain and exiting the snaking region. On the other hand, unequally spaced two-convecton states are expected to lie on nested isolas (Burke \& Knobloch 2009; Knobloch et al. 2010); on the real line there will be an infinite number of such isolas, corresponding to the infinite number of discrete separations permitted by the locking between the oscillatory tails of neighbouring convectons. Moreover, each set of nested isolas corresponds to a fixed number of rolls within the two convectons forming the two-pulse state. Thus, on the real line the snaking region is expected to contain an infinite stack of such isolas, each corresponding to a bound state of convectons with different number of rolls (Burke \& Knobloch 2009; Knobloch et al. 2010). In general, the breakup of the snakes-and-ladders structure of the pinning region (Burke \& Knobloch 2007a) that gives rise to these structures becomes exponentially small as soon as the two convectons are substantially far apart. This is a consequence of the exponential dependence on the separation of the effective interaction force between pairs of convectons. This makes it impractical to look for multipulse states in large domains. For $\Gamma=14$, however, such states can be located and the effects of non-PBC investigated.

In the following, we focus on the case of two equidistant convectons and refer to the individual convectons using their spatial phase (Burke \& Knobloch 2007a). Evenparity convectons have phase $0(\pi)$ if the fluid is rising (descending) in the centre. Odd-parity convectons have phase $\pi / 2(3 \pi / 2)$ if the odd roll in the centre rotates anticlockwise (clockwise). Thus, the convectons in figure $1(a, d)$ have phases $\pi, 3 \pi / 2$, respectively. In this terminology, the (equidistant) two-pulse states just discussed are of type $(0,0)$ or $(\pi, \pi)$ (even parity) and $(\pi / 2, \pi / 2)$ or $(3 \pi / 2,3 \pi / 2)$ (odd parity). As for single-pulse states this situation is unfolded when the boundary conditions are changed to NBC.

In figure 10, we show the effect of this change on even-parity two-pulse states. Figure 10 $(a)$ shows two-pulse states composed of identical even-parity convectons. The branch labelled $2 \mathrm{PC}_{12}$ is of type $(0,0)$ and consists of an even-parity central convecton with a rising warm plume in the centre and a pair of wall-attached states at either end of the domain (figure 11a, top). Glued together back-to-back, the latter represent another even convecton with a rising plume in the centre. Thus, the observed structure is in fact identical to the $(0,0)$ two-pulse configuration that one 

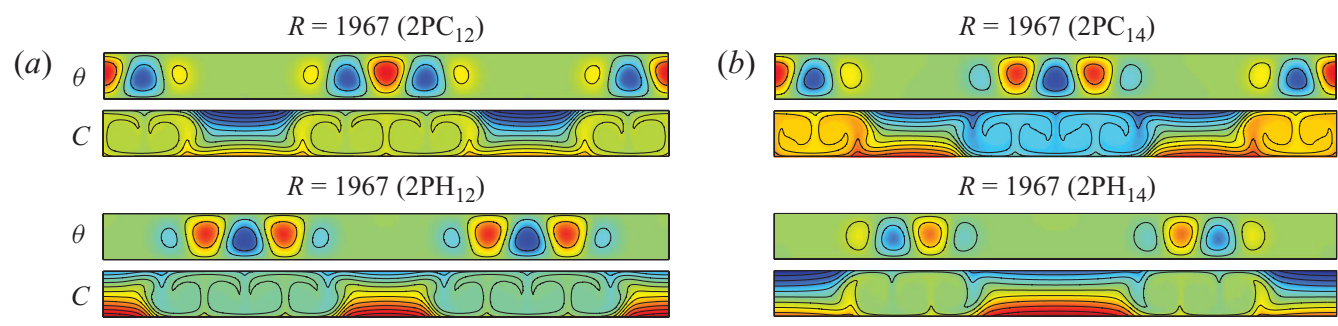

FIGURE 11. (Colour online) Even-parity two-pulse states in a $\Gamma=14$ domain. (a) Solutions based on 12 rolls: bound states $2 \mathrm{PC}_{12}$ (top) and $2 \mathrm{PH}_{12}$ (bottom), both at $R=1967$. The solutions are related by a hidden symmetry and so fall on the same branch in figure $10(a)$. (b) Solutions based on 14 rolls: bound state $2 \mathrm{PC}_{14}$ (top) at $R=1967$ and bound state $2 \mathrm{PH}_{14}$ (bottom) at $R=2015$.

finds with $\mathrm{PBC}$ on the domain $\Gamma$. Of course, the snaking region is not nearly as well developed since each convecton only has the length $\Gamma / 2$ available for growth. In contrast, the branch labelled $2 \mathrm{PH}_{12}$ consists of $(\pi, \pi)$ states with the convectons located on either side of a central hole (figure 11a, bottom). As before, these solutions are phase-matched and hence terminate together, this time on the branch $\mathrm{SOC}_{12}$ with 12 rolls in the domain $\Gamma$. However, in addition both states are even under reflection in $x=\Gamma / 4$ and satisfy $\mathrm{NBC}$ on the half-domain $0 \leqslant x \leqslant \Gamma / 2$. The discussion in $\S 3.1$ for single even-parity convectons with $\mathrm{NBC}$ therefore applies and shows that the two states in figure $11(a)$ are related by a (hidden) symmetry and hence lie on the same branch; cf. figure $10(a)$ and figure $2(a)$. This is no longer the case for the $2 \mathrm{PC}_{14}$ and $2 \mathrm{PH}_{14}$ states shown in figure $10(b)$. The states on the branch labelled $2 \mathrm{PC}_{14}$ also consist of an even-parity central convecton, this time with a descending cold plume in the centre, together with a pair of wall-attached states at either end of the domain which when glued together again represent an even convecton with a rising plume in the centre (figure $11 b$, top). Thus, the structure shown is in fact identical to the $(0, \pi)$ two-pulse configuration that one finds with $\mathrm{PBC}$ on the domain $\Gamma$. In contrast, the states on the $2 \mathrm{PH}_{14}$ branch correspond to bound states of pairs of back-to-back odd-parity convectons in configuration $(\pi / 2,3 \pi / 2)$ (figure $11 b$, bottom). The resulting structure is also even about the middle of the domain and hence also identical to a two-pulse state with $\mathrm{PBC}$ on the domain $\Gamma$. The $2 \mathrm{PC}_{14}$ and $2 \mathrm{PH}_{14}$ in figure $11(b)$ are phase-matched and hence also terminate together, this time on the branch $\mathrm{SOC}_{14}$ of 14 rolls in the domain $\Gamma$. Once again, both states satisfy NBC on the half-domain $0 \leqslant x \leqslant \Gamma / 2$, but this time both states are odd under reflection in $x=\Gamma / 4$. In this case, the discussion of $\S 3.3$ shows that the two states in figure $11(b)$ are not symmetry-related and hence generically lie on distinct solution branches, as seen in figure $10(b)$.

The question now arises as to the presence of two-pulse states with overall odd parity, i.e. with overall point symmetry. Such states are indeed present and bifurcate, much as the single-pulse states, from the $\mathrm{SOC}_{13}$ and $\mathrm{SOC}_{15}$ branches. Figure $12(a)$ shows that two distinct branches of two-pulse states bifurcate simultaneously from $\mathrm{SOC}_{13}$. The first, referred to as $2 \mathrm{PC}_{13}$, consists of a central convecton with the second convecton split between the two lateral walls (figure 13a, top). The central convecton has odd parity but the remaining convecton that is split between the boundaries cannot be glued together to form a second odd-parity convecton. This is because the $2 \mathrm{PC}_{13}$ state is in fact point-symmetric. This is so for the state labelled $2 \mathrm{PH}_{13}$ as well. This state consists of a central hole with a pair of convectons on either side. In figure 13( $a$, middle and bottom), this state resembles a bound state of $(0, \pi)$ type located at $x= \pm \Gamma / 4$. 
(a)

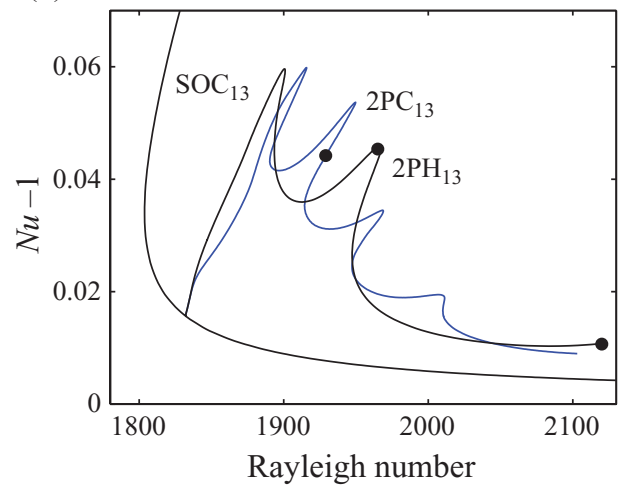

(b)

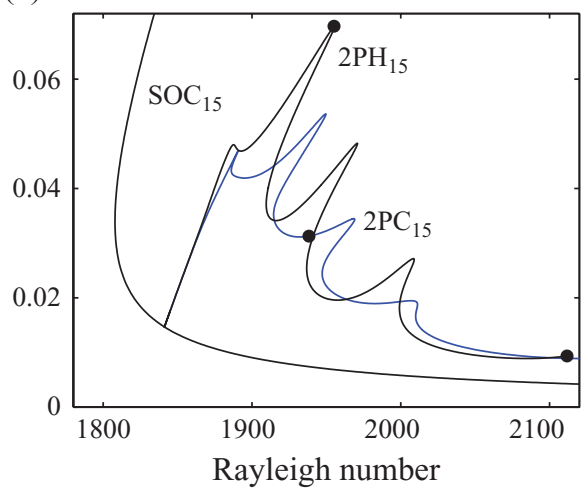

Figure 12. (Colour online) Bifurcation diagrams for two-pulse point-symmetric states in a $\Gamma=14$ domain. (a) Solutions based on 13 rolls. (b) Solutions based on 15 rolls. Solid dots indicate the locations of the solutions shown in figure 13.

(a)

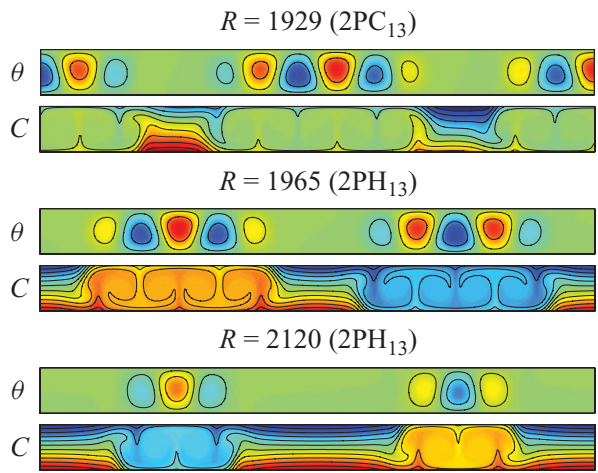

(b)

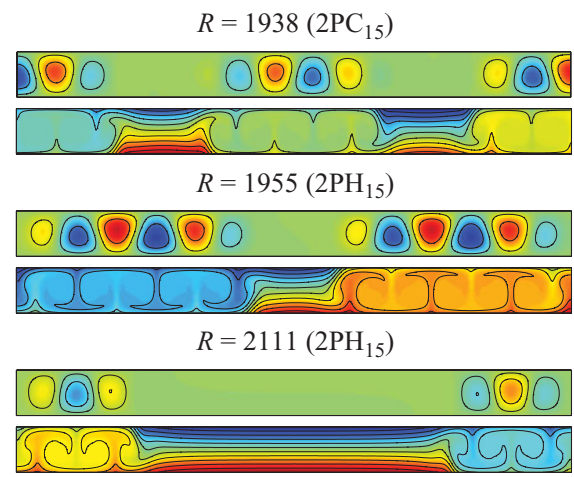

FIgURE 13. (Colour online) Point-symmetric two-pulse states in a $\Gamma=14$ domain. (a) Solutions based on 13 rolls: bound state $2 \mathrm{PC}_{13}$ at $R=1929$ (top), bound state $2 \mathrm{PH}_{13}$ at $R=1965$ (middle) and bound state $2 \mathrm{PH}_{13}$ at $R=2120$ (bottom). (b) Solutions based on 15 rolls: bound state $2 \mathrm{PC}_{15}$ at $R=1938$ (top), bound state $2 \mathrm{PH}_{15}$ at $R=1955$ (middle) and bound state $2 \mathrm{PH}_{15}$ at $R=2111$ (bottom).

However, the point symmetry implies that the boundary conditions on the right convecton are Dirichlet boundary conditions (DBC) at $x=0$ and $\mathrm{NBC}$ at $x=\Gamma / 2$, and similarly for the left convecton. Thus, neither convecton has exact even parity. This is a consequence of their mutual interaction. Indeed, in this example the state obtained by reflecting it in the boundary $x=\Gamma / 2$ differs from a $\Gamma / 2$ translation, in contrast to the situation in figure 10 , i.e. the state $2 \mathrm{PH}_{13}$, like $2 \mathrm{PC}_{13}$, does not satisfy $\mathrm{PBC}$ with period $\Gamma$. Moreover, since the $2 \mathrm{PH}_{13}$ state is not a bound state of two pure parity states, it changes its appearance along the branch. For example, near the bifurcation point on $\mathrm{SOC}_{13}$, it no longer looks like a bound state of two even convectons and resembles instead an extended point-symmetric state with a defect at $x=0$ (not shown). Of course this must be so since the $\mathrm{SOC}_{13}$ state is point-symmetric.

Similar but different two-pulse states also bifurcate from $\mathrm{SOC}_{15}$ (figure $12 b$ ). The states, labelled $2 \mathrm{PC}_{15}$ and $2 \mathrm{PH}_{15}$, are shown in figure $13(b)$. Both are point-symmetric, with $2 \mathrm{PC}_{15}$ consisting of a central point-symmetric convecton with two wall-attached convectons at either wall (top) while $2 \mathrm{PH}_{15}$ consists of a central hole surrounded by apparently odd-parity convectons on either side (middle). However, since each 


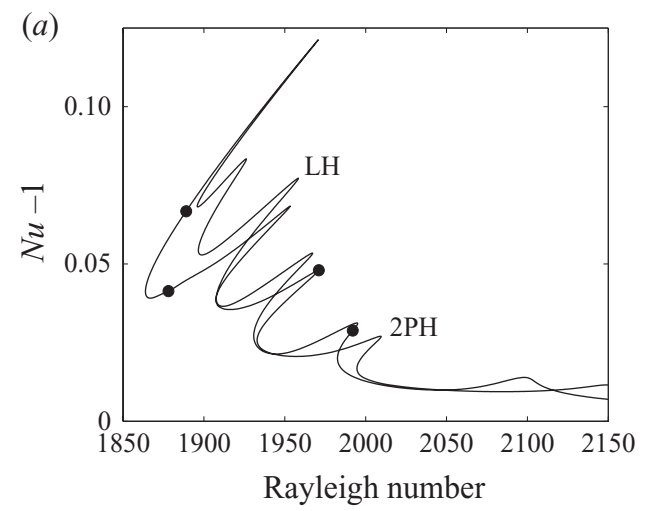

(b)

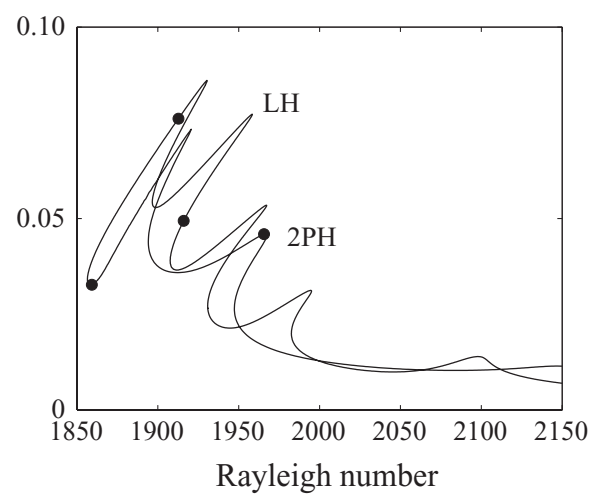

Figure 14. Bifurcation diagram with ICCBC in a $\Gamma=14$ domain showing $(a)$ a branch of odd-parity states with both single-pulse hole states $(\mathrm{LH})$ and two-pulse holes states $(2 \mathrm{PH})$, and $(b)$ a branch of even-parity states with both single-pulse hole states ( $\mathrm{LH})$ and two-pulse holes states $(2 \mathrm{PH})$. In both cases, the two solution types lie on a single curve with the transition between them near the leftmost saddle-node (figures $15 b$ and $16 b$ ).

constituent convecton satisfies $\mathrm{NBC}$ on one side and $\mathrm{DBC}$ on the other, neither is a pure parity state. As a result, the form of these solutions changes dramatically as one follows each branch. Figure $13\left(b\right.$, bottom) shows a solution on the $2 \mathrm{PH}_{15}$ branch far from the bifurcation on the $\mathrm{SOC}_{15}$ branch, and reveals that the solution that resembled a bound state of two convectons of type $(3 \pi / 2,3 \pi / 2)$ at $R=1955$ becomes at larger $R$ a pair of out-of-phase states confined to the vicinity of either boundary. Thus, in contrast to figure $13(a)$ the distance between the two convectons is a strong function of location along the $2 \mathrm{PH}_{15}$ branch. This is a consequence of strongly inhomogeneous growth of individual convectons near NBC boundaries. Such behaviour resembles closely recent results on the Swift-Hohenberg equation with quadratic-cubic (SH23) and cubic-quintic (SH35) nonlinearities (Dawes 2009; Houghton \& Knobloch 2009; Kozyreff et al. 2009), which clearly demonstrate that the loss of translation invariance resulting from changing $\mathrm{PBC}$ to $\mathrm{NBC}$ has a dramatic effect on the states present with PBC that do not satisfy the new boundary conditions. In particular, one finds that when this is the case the solution wavenumber is no longer well defined, and the wavelength and in particular the location of the localized structures varies substantially along the corresponding solution branches.

\section{Two-convecton states: closed container boundary conditions}

The use of ICCBC in place of NBC does not change the basic symmetries of the problem. Consequently, we expect the same broad class of solutions in both cases. Of course the use of ICCBC destroys the SOC states whose place is now taken by large-amplitude extended states resembling the mixed-mode states present with PBC. Thus, while we continue to expect snaking we also expect the snaking branch to turn continuously into a mixed-mode-like state at large amplitude, much as found in $\S 4$ for single-pulse states. In fact, the situation is rather more complicated.

Figure 14(a) shows the resulting bifurcation diagram for odd-parity (pointsymmetric) states. The figure shows the locations of two-pulse states with a hole at $x=0(2 \mathrm{PH})$ and of hole-like states with a wall-attached state on either side (LH), and reveals that these two states are now found on the same solution branch. 
(a)

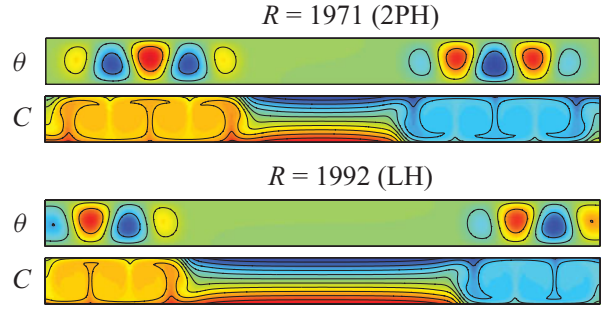

(b)

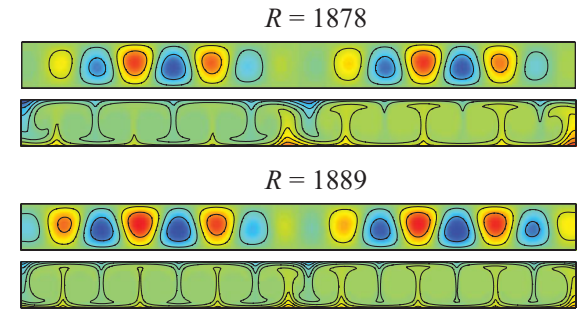

FiguRE 15. (Colour online) (a) An odd-parity state at $R=1971$, labelled 2PH in figure 14(a), together with a hole-like state at $R=1992$, labelled LH. $(b)$ Two solutions near the leftmost saddle-node in figure 14(a) showing the transition between the $2 \mathrm{PH}$ and $\mathrm{LH}$ states.

Thus, with ICCBC the solution branch passes through the snaking region twice, once going up and once coming down. This type of reconnection is familiar from the Swift-Hohenberg equation with non-NBC (Houghton \& Knobloch 2009) and is known to leave behind disconnected large amplitude states which we do not calculate.

In figure $15(a)$, we show sample solutions from the $2 \mathrm{PH}$ (top) and $\mathrm{LH}$ (bottom) parts of the branch. The solutions are qualitatively similar but in the hole solution the hole is bounded by two wall states attached to either boundary. In contrast, in the two-pulse state, the pulses do not reach the walls and only settle towards the wall when the two-pulse state turns into a hole state. The location of this (gradual) transition is shown in figure $15(b)$. We see that as one approaches the leftmost saddle-node along the two-pulse branch, each convecton starts to grow in a markedly asymmetric fashion, unable to add rolls near the boundary while continuing to add rolls in the middle of the domain and almost filling in the hole in the centre. Near the leftmost saddle-node, the resulting two-pulse state adds an extra roll near each wall, thereby becoming a hole state. Thus, the two-pulse branch turns continuously into a branch of hole states. As one follows the branch further, the hole broadens by eliminating rolls on either side while maintaining the odd parity of the state (figure 15a, bottom). Evidently, the asymmetric growth of the convectons in the transition region is a consequence of interaction with the boundary or equivalently with an image convecton that is nearer than the other convecton comprising the state. We mention that the two-pulse state in figure $15(b)$ at $R=1878$ closely resembles the $R=1955$ state in figure $13(b)$. This is simply a consequence of the fact that the convectons are still sufficiently far from the lateral walls for the details of the boundary conditions to be reflected in the solution. Indeed, one finds that prior to the transition to the hole branch, the $2 \mathrm{PH}$ branch in figure $14(a)$ is almost identical to the $2 \mathrm{PH}_{15}$ branch in figure $12(b)$. The differences between these branches are confined to the left saddle-nodes where the nucleating rolls are weak and the different boundary conditions are able to exert a noticeable effect.

The corresponding results for even-parity states are shown in figures $14(b)$ and 16 . Once again, the two-pulse and hole states fall on the same solution branch with a continuous transition between them in the vicinity of the leftmost saddle-node. As in the odd-parity case the transition region between the two-pulse and hole states is characterized by markedly asymmetric growth of the convectons once these reach close to the lateral walls. In this case, the two-pulse branch in figure $14(b)$ is of $\mathrm{H}$-type and very similar to the branch $2 \mathrm{PH}_{12}$ in figure $10(a)$. Indeed, the solutions in figure $16(a$, top) and figure $11(a$, bottom) are very similar. This is a consequence of the fact that at this parameter value the convectons are still far from the wall. 
(a)

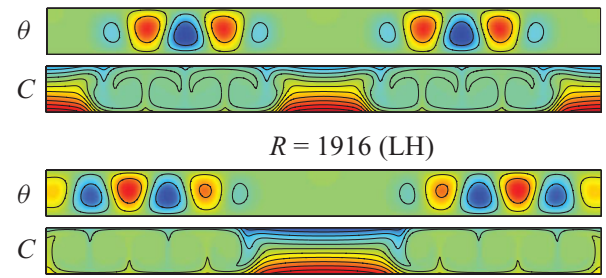

(b)

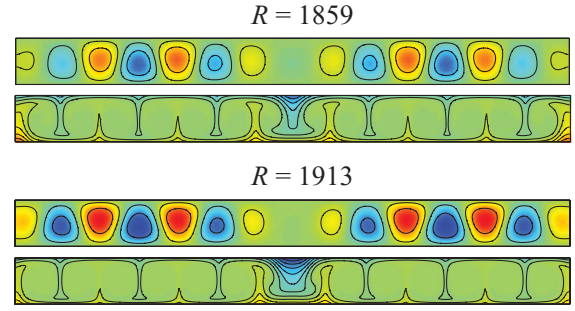

FIGURE 16. (Colour online) ( $a$ ) An even-parity state at $R=1966$, labelled $2 \mathrm{PH}$ in figure 14(b), together with a hole-like state at $R=1916$, labelled LH. $(b)$ Two solutions near the leftmost saddle-node in figure $14(b)$ showing the transition between the $2 \mathrm{PH}$ and $\mathrm{LH}$ states.

\section{Conclusions}

In this paper, we have examined the existence and properties of steady spatially localized structures in binary fluid convection in a two-dimensional laterally bounded domain. We examined two types of boundary conditions, NBC and ICCBC. The former boundary conditions are convenient since they select from among the states satisfying $\mathrm{PBC}$, a class of states that satisfy $\mathrm{NBC}$ without perturbing these states. This fact follows from a general construction that allows one to embed solutions with NBC on a domain $\Gamma$ among solutions with PBC on a domain with period $2 \Gamma$. This construction carries over to a $\Gamma$ domain with $\mathrm{PBC}$ when the state is even, but not for odd-parity states. Thus, when $\Gamma=14$, even-parity states, whether single-pulse convectons or anticonvectons, or two-pulse states with overall even parity, behave in much the same way as periodic states on a $\Gamma$ domain: all snake within the snaking or pinning region in Rayleigh number, and once the domain is almost full they exit the snaking region towards the left and terminate on a branch consisting of an even number of rolls within $\Gamma$ (either $\mathrm{SOC}_{12}$ or $\mathrm{SOC}_{14}$ ) or equivalently a branch consisting of six $\left(\mathrm{P}_{6}\right)$ or seven $\left(\mathrm{P}_{7}\right)$ pairs of rolls within $\Gamma$. We conjecture that the snaking region for all these states is the same and is defined by the first and last tangencies between the unstable manifold of the conduction state and the centrestable manifold of periodic convection (Woods \& Champneys 1999; Coullet et al. 2000; Beck et al. 2009). However, the situation for odd-parity states is more complex. Here the NBC and periodic states are no longer the same. In addition, odd-parity states pump concentration from one side of the structure to the other (Batiste et al. 2006) although their point symmetry guarantees that they remain stationary. Since the odd-parity states with NBC are distinct states, their behaviour will differ from that of periodic states, and we have identified two different types of single-pulse states that snake, convectons and anticonvectons (holes). Since these states are no longer related by translation, they terminate on different extended states, in our case $\mathrm{SOC}_{13}$ and $\mathrm{SOC}_{15}$, respectively, together with a new class of states we call mixed modes which do not snake. Since the mixed modes by definition contain a defect or hole, they are often difficult to distinguish from hole states (or from a convecton state with holes at the sidewalls) unless one understands their location in the bifurcation diagram.

There is, however, an analogue of a single-pulse even-parity state on the domain $2 \Gamma$ that survives in a $\Gamma$ domain with NBC. This is the wall state. This state can be thought of as a half-convecton attached to one or other lateral wall. These wall states also snake and with each turn add a single roll in $\Gamma$. Thus, they grow half as fast as a convecton in a $\Gamma$ domain and must execute twice as many turns before the domain 
is full and the wall states terminate on an extended state. This type of state persists with ICCBC.

The situation is yet more interesting for odd-parity multipulse states, and in particular for the two-pulse states considered here. These states also bifurcate from $\mathrm{SOC}_{13}$ and $\mathrm{SOC}_{15}$ but do so further from the saddle-node than the single-pulse states. These states also snake, and in larger domains snake in the same snaking region as even-parity states. This is because the boundary conditions suppress horizontal concentration pumping by odd-parity states (Mercader et al. 2010a). Moreover, in the presence of ICCBC, these odd-parity states no longer have a well-defined wavenumber and so change their appearance and location in the domain as their amplitude or the Rayleigh number varies. Thus, these states can resemble a two-pulse state in a part of the parameter range and a pair of wall states in another part.

This type of state transformation occurs continuously, without bifurcation, and closely resembles similar behaviour already observed in the Swift-Hohenberg equation (Dawes 2009; Houghton \& Knobloch 2009). We believe that this type of behaviour is responsible for the observation of similar states in natural doubly diffusive convection in a vertical cavity (Ghorayeb \& Mojtabi 1997). This system is similar to the present one with the vertical direction representing the extended direction, although it lacks the analogue of the $z \rightarrow 1-z$ symmetry. Multiconvectons have also been studied in binary mixtures in a porous medium (Lo Jacono, Bergeon \& Knobloch 2010) but only with PBC. This system shares the symmetries of the system studied here but is simpler since the velocity solves a time-independent equation and all velocity boundary conditions are necessarily stress-free.

It is of interest finally to ascertain the origin of the variety of states identified here for realistic parameter values and boundary conditions. To this end, we have performed homotopic continuation from the stress-free boundary conditions required by NBC to the no-slip ICCBC. For this purpose, we write

$$
\beta w \pm(1-\beta) w_{x}=0 \quad \text { at } \quad x= \pm \Gamma / 2,
$$

and leave the remaining boundary conditions unchanged. Here $\beta$ represents a homotopy parameter with $\beta=0$ corresponding to $\mathrm{NBC}$ and $\beta=1$ corresponding to ICCBC. Figure 17 shows the different types of reconnection experienced by oddparity states. Figure $17(a)$ describes the breakup of the NBC $(\beta=0)$ bifurcation diagrams in figures $6(a)$ and $12(a)$ when $\beta=0.05$, and shows that the snaking singlepulse solutions (solid curve) now connect smoothly to large-amplitude domain-filling states with 13 cells, much as in figure $9(b)$ with ICCBC. These states are all of C-type, i.e. all convect in the centre of the domain with convection suppressed near the lateral boundaries. At the same time, the large-amplitude odd-parity mixed modes now connect to a snaking branch of two-pulse states and do so via an interval of SOC-like states (dashed curve). Of these states the mixed modes have a hole in the centre of the domain as do the two-pulse states, i.e. the mixed modes are of H-type while the two-pulse states are of 2PH-type. However, the SOC-like states consist of states with convection in the centre (C-type), indicating that with non-Neumann boundary conditions the presence or absence of convection at $x=0$ is no longer a property that can be used to characterize a given solution branch; cf. Houghton \& Knobloch (2009). Figure 17(a) also reveals the presence of small-amplitude three-pulse states (dash-dotted curve) connected to two-pulse states of 2PC-type via a portion of the original $\mathrm{SOC}_{13}$ branch. All of these states convect in the centre of the domain and so are of C-type. 

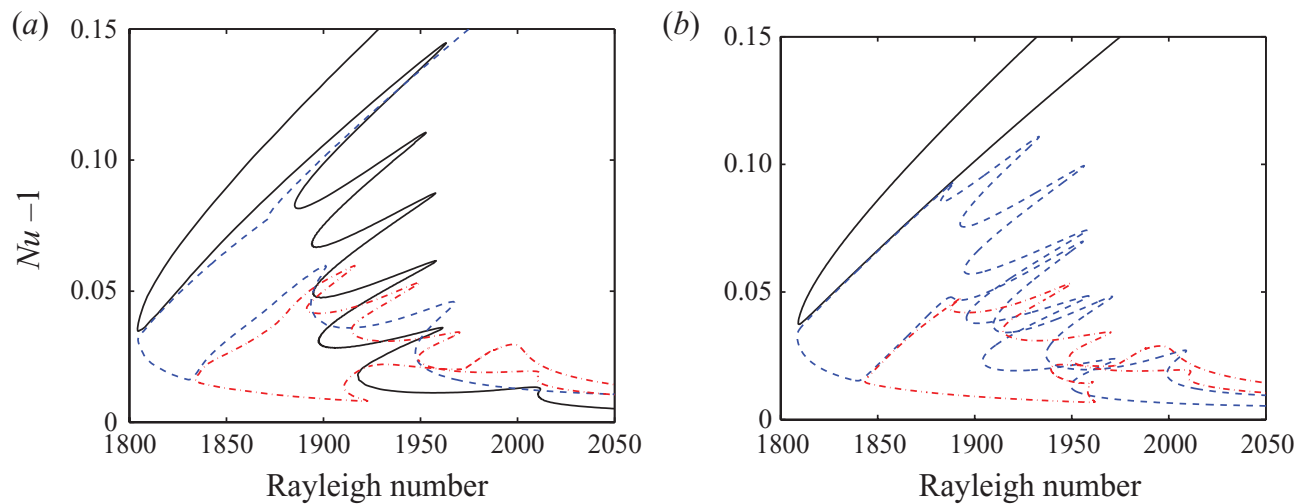

FIGURE 17. (Colour online) Bifurcation diagrams for odd-parity convectons when $\beta=0.05$, with the remaining boundary conditions unchanged. The breakup of $(a)$ the $\beta=0$ bifurcation diagrams in figures $6(a)$ and $12(a)$, and $(b)$ the $\beta=0$ bifurcation diagrams in figures $6(b)$ and $12(b)$. The branches are distinguished by the maximum number $n$ of pulses present along the branch: $n=1$ (solid curves), $n=2$ (dashed curves), $n=3$ (dash-dotted curves).

Figure 17(b) describes the corresponding breakup of the NBC $(\beta=0)$ diagrams in figures $6(b)$ and $12(b)$. In this case, the large-amplitude $\mathrm{SOC}_{15}$ state with convection in the centre connects to the corresponding C-type mixed-mode state, while the snaking hole branch in figure 6(b) connects to small amplitude C-type 15 cell states which in turn connect to two-pulse states arising from $2 \mathrm{PH}_{15}$ in figure $12(b)$. Thus, in this case too the reconnection leads to a branch containing both H-type and C-type states. In addition, the states identified as $2 \mathrm{PC}_{15}$ in figure $12(b)$ connect to a branch of three-pulse states, again via an interval of small amplitude 15 cell states. All of these states are of C-type.

It follows that in both cases the homotopic continuation unfolds the pitchfork bifurcations on the SOC branch corresponding to Eckhaus bifurcations (Bergeon et al. 2008). It is likely that this breakup process affects all Eckhaus points in like fashion, creating connections between states with $n-1$ and $n$ convectons, $n=1,2, \ldots$. With increasing $\beta$ the single-pulse curve (solid line) in figure 17(a) morphs into the single-pulse ICCBC branch in figure $9(a)$, while the dashed curve in figure $17(b)$ morphs into the ICCBC branch shown in figure 14(a). Similar behaviour is found for even-parity states as well (not shown).

In this paper, we have not discussed in detail the stability of these states, although we know that many are stable (Batiste et al. 2006). Likewise, the states found by Ghorayeb $\&$ Mojtabi (1997) were computed using time-stepping suggesting that they are also all stable. At present, there is no experimental confirmation of our results, although some experiments on water-ethanol mixtures (Kolodner 1993) have identified stationary localized states embedded in a background of small-amplitude travelling waves. We believe that the background waves are present because the Rayleigh number used exceeded the threshold for absolute instability of the conduction state, and predict that if this were lowered below this threshold, steady localized convectons embedded in a laminar background would be observed (Alonso et al. 2007).

This work was supported in part by DGICYT under grant FIS2009-08821 and by the National Science Foundation under grant DMS-0908102. 


\section{REFERENCES}

Alonso, A., Batiste, O., Knobloch, E. \& Mercader, I. 2010 Convectons. In Localized States in Physics: Solitons and Patterns (ed. O. Descalzi, M. G. Clerc, S. Residori \& G. Assanto), pp. 109-126. Springer.

Alonso, A., Batiste, O., Meseguer, A. \& Mercader, I. 2007 Complex dynamical states in binary mixture convection with weak negative Soret coupling. Phys. Rev. E 75, 026310 (1-15).

BARTEN, W., LÜCKe, M., KAMPs, M. \& SChMitZ, R. 1995 Convection in binary mixtures. II. Localized traveling waves. Phys. Rev. E 51, 5662-5680.

Batiste, O. \& KNobloch, E. $2005 a$ Simulations of localized states of stationary convection in ${ }^{3} \mathrm{He}-{ }^{4} \mathrm{He}$ mixtures. Phys. Rev. Lett. 95, 244501 (1-4).

Batiste, O. \& KNOBloch, E. $2005 b$ Simulations of oscillatory convection in ${ }^{3} \mathrm{He}-{ }^{4} \mathrm{He}$ mixtures in moderate aspect ratio containers. Phys. Fluids 17, 064102 (1-14).

Batiste, O., Knobloch, E., Alonso, A. \& Mercader, I. 2006 Spatially localized binary-fluid convection. J. Fluid Mech. 560, 149-158.

Beck, M., Knobloch, J., Lloyd, D. J. B., SAndstede, B. \& Wagenknecht, T. 2009 Snakes, ladders and isolas of localized patterns. SIAM J. Math. Anal. 41, 936-972.

Bergeon, A., Burke, J., Knobloch, E. \& Mercader, I. 2008 Eckhaus instability and homoclinic snaking. Phys. Rev. E 78, 046201 (1-16).

Bergeon, A. \& KNOBLOCH, E. 2008 Spatially localized states in natural doubly diffusive convection. Phys. Fluids 20, $034102(1-8)$.

Blanchflower, S. 1999 Magnetohydrodynamic convectons. Phys. Lett. A 261, 74-81.

Blanchflower, S. \& Weiss, N. 2002 Three-dimensional magnetohydrodynamic convectons. Phys. Lett. A 294, 297-303.

Burke, J. \& KNobloch, E. 2007a Snakes and ladders: localized states in the Swift-Hohenberg equation. Phys. Lett. A 360, 681-688.

BuRKe, J. \& KNOBLOCH, E. $2007 b$ Homoclinic snaking: structure and stability. Chaos 17, 037102 $(1-15)$.

Burke, J. \& Knobloch, E. 2009 Multipulse states in the Swift-Hohenberg equation. Discrete Contin. Dyn. Syst. Suppl. 109-117.

Champneys, A. R. 1998 Homoclinic orbits in reversible systems and their applications in mechanics, fluids and optics. Physica D 112, 158-186.

Coullet, P., Riera, C. \& Tresser, C. 2000 Stable static localized structures in one dimension. Phys. Rev. Lett. 84, 3069-3072.

Crawford, J. D., Golubitsky, M., Gomes, M. G. M., Knobloch, E. \& Stewart, I. N. 1991 Boundary conditions as symmetry constraints. In Singularity Theory and Its Applications, Warwick 1989, Part II (ed. M. Roberts \& I. Stewart), Lecture Notes in Mathematics 1463, pp. 63-79. Springer.

Crawford, J. D. \& KNOBLOCh, E. 1991 Symmetry and symmetry-breaking bifurcations in fluid mechanics. Annu. Rev. Fluid Mech. 23, 341-387.

DaweS, J. H. P. 2009 Modulated and localized states in a finite domain. SIAM J. Appl. Dyn. Syst. 8, 909-930.

Ghorayeb, K. \& Mojtabi, A. 1997 Double diffusive convection in a vertical rectangular cavity. Phys. Fluids 9, 2339-2348.

van der Heijden, G. H. M., Champneys, A. R. \& Thompson, J. M. T. 2002 Spatially complex localisation in twisted elastic rods constrained to a cylinder. Intl J. Solids Struct. 39, 1863-1883.

Houghton, S. M. \& Knobloch, E. 2009 Homoclinic snaking in bounded domains. Phys. Rev. E 80, $026210(1-15)$.

Hunt, G. W., Peletier, M. A., Champneys, A. R., Woods, P. D., Ahmer Wadee, M., Budd, C. J. \& LoRD, G. J. 2000 Cellular buckling in long structures. Nonlinear Dyn. 21, 3-29.

JUNG, D. \& LÜCKE, M. 2007 Bistability of moving and self-pinned fronts of supercritical localized convection structures. Europhys. Lett. 80, 14002 (1-6).

Knobloch, J., Lloyd, D. J. B., Sandstede, B. \& Wagenknecht, T. 2010 Isolas of 2-pulse solutions in homoclinic snaking scenarios. J. Dyn. Diff. Eqs. (in press).

Kolodner, P. 1993 Coexisting traveling waves and steady rolls in binary-fluid convection. Phys. Rev. E 48, R665-R668. 
Kolodner, P., Glazier, J. A. \& Williams, H. 1990 Dispersive chaos in one-dimensional travelingwave convection. Phys. Rev. Lett. 65, 1579-1582.

Kolodner, P., Surko, C. M. \& Williams, H. 1989 Dynamics of traveling waves near onset of convection in binary fluid mixtures. Physica D 37, 319-333.

KozyrefF, G., Assemat, P. \& Chapman, S. J. 2009 Influence of boundaries on localized patterns. Phys. Rev. Lett. 103, 164501 (1-4).

Lee, K. J., McCormick, W. D., Pearson, J. E. \& Swinney, H. L. 1994 Experimental observation of self-replicating spots in a reaction-diffusion system. Nature 369, 215-218.

Lioubashevski, O., Hamiel, Y., Agnon, A., Reches, Z. \& Fineberg, J. 1999 Oscillons and propagating solitary waves in a vertically vibrated colloidal suspension. Phys. Rev. Lett. 83, 3190-3193.

Lo Jacono, D., Bergeon, A. \& Knobloch, E. 2010 Spatially localized binary fluid convection in a porous medium. Phys. Fluids 22, 073601 (1-13).

Mercader, I., Alonso, A. \& Batiste, O. 2004 Numerical analysis of the Eckhaus instability in travelling-wave convection in binary mixtures. Eur. Phys. J. E 15, 311-318.

Mercader, I., Batiste, O. \& Alonso, A. 2006 Continuation of travelling-wave solutions of the Navier-Stokes equations. Intl J. Numer. Methods Fluids 52, 707-721.

Mercader, I., Batiste, O., Alonso, A. \& Knobloch, E. 2009 Localized pinning states in closed containers: homoclinic snaking without bistability. Phys. Rev. E 80, 025201(R)-1-4.

Mercader, I., Batiste, O., Alonso, A. \& Knobloch, E. $2010 a$ Convectons in periodic and bounded domains. Fluid Dyn. Res. 42, 025505 (1-10).

Mercader, I., Batiste, O., Alonso, A. \& Knobloch, E. $2010 b$ Dissipative solitons in binary fluid convection. Discrete Contin. Dyn. Syst. Suppl. (in press).

PomeaU, Y. 1986 Front motion, metastability and subcritical bifurcations in hydrodynamics. Physica D 23, 3-11.

RichteR, R. \& BARASHENKov, I. V. 2005 Two-dimensional solitons on the surface of magnetic fluids. Phys. Rev. Lett. 94, 184503 (1-4).

SCHNEIDER, T. M., GibSON, J. F. \& BuRke, J. 2010 Snakes and ladders: localized solutions of plane Couette flow. Phys. Rev. Lett. 104, 104501 (1-4).

Steinberg, V., Fineberg, J., Moses, E. \& Rehberg, I. 1989 Pattern selection and transition to turbulence in propagating waves. Physica D 37, 359-383.

Umbanhowar, P. B., Melo, F. \& Swinney, H. L. 1996 Localized excitations in a vertically vibrated granular layer. Nature 382, 793-796.

Vladimirov, A. G., McSloy, J. M., Skryabin, D. V. \& Firth, W. J. 2002 Two-dimensional clusters of solitary structures in driven optical cavities. Phys. Rev. E 65, 046606 (1-11).

Wadee, M. K., Coman, C. D. \& Bassom, A. P. 2002 Solitary wave interaction phenomena in a strut buckling model incorporating restabilisation. Physica D 163, 26-48.

Woods, P. D. \& Champneys, A. R. 1999 Heteroclinic tangles and homoclinic snaking in an unfolding of a degenerate Hamiltonian-Hopf bifurcation. Physica D 129, 147-170. 